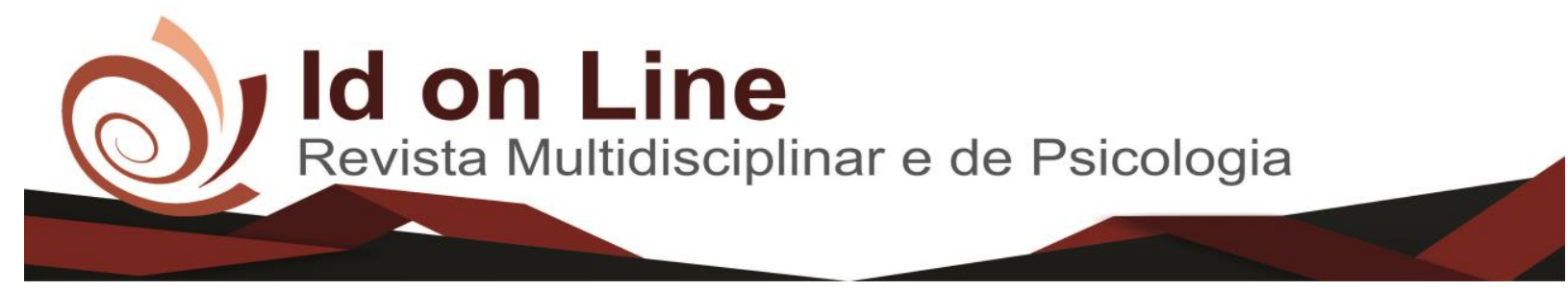

Artigo

\title{
Análise Parasitológica de contaminantes de origem fecal encontrados em uma Creche do interior da Bahia
}

\author{
Naysnan Rocha Brito Almeida ${ }^{1}$ Alana Soares Carvalho ${ }^{2}$ Camila Amaral Moreno ${ }^{3}$
}

\begin{abstract}
Resumo: O parasitismo intestinal ainda é considerado um grave problema de saúde pública, estima-se que mais de 2 bilhões de pessoas estejam infectadas por parasitas intestinais, dentre eles destaca-se: Ascaris lumbricoides, Trichuris trichiura, Necator americanus e Ancylostoma duodenale. Dentre os grupos populacionais, as crianças são as mais acometidas pelas parasitoses. A maior concentração dos casos de parasitismo ocorre em áreas onde o saneamento básico é inexistente ou insuficiente, características de países em desenvolvimento com inadequada infraestrutura sanitária e ambiental. Este trabalho tem por objetivo identificar e avaliar o perfil epidemiológico de contaminantes de origem fecal, presentes em fômites de uma creche no interior da Bahia, em um distrito do município de Planalto. Trata-se de um estudo, descritivo exploratório com abordagem qualitativa. O local escolhido para realização do estudo foi uma creche municipal. $\mathrm{O}$ trabalho foi realizado a partir de coleta de amostras presentes no interior da creche, com base no método de Graham. Os dados foram organizados e tabulados com auxílio do programa Microsoft Excel 2010. Os resultados apontaram 87,5\% de amostras positivas e 12,5\% negativas. Conclui-se que a incidência de parasitas em creches é um problema de saúde pública de alta magnitude que merece atenção especial.
\end{abstract}

Palavras-chave: Contaminação, Crianças, Helmintos

\section{Parasitological Analysis of fecal contamination origins in a Nursery in the interior of Bahia}

\begin{abstract}
The Intestinal parasitism is still considered a serious public health problem, it's estimated that over 2 million people are infected by intestinal parasites, among them standsout: Ascaris lumbricoides, Trichuris trichiura, Necator americanus e Ancylostoma duodenale. Among the population groups, children are the most affected by the parasitic diseases. The highest parasitism concentration occurs in places where basic sanitation is non - existent or inefficiency, characteristics from developing countries with inadequate environmental and sanitary infrastructure. This study aims to identify and analysis the epidemiological profile fecal origin contaminants, presents in things from a day care center in the interior of Bahia, at a district from the municipality of Planalto. It's a exploratory descriptive study with qualitative approach. The local chosen for conducting the study was a day care center. The work was realized by sample collection presents inside of the day care center, based on Graham's method. The dates were organized and tabulated with the aid of Microsoft excel 2010 program.The results pointed $87,5 \%$ positive samples and $12,5 \%$ negative samples. Concludesthat the parasites incidence in day care centers is a high magnitude public health problem that deserves special attention
\end{abstract}

Keywords: Contamination, Children,Helminths.

\footnotetext{
${ }^{1}$ Graduanda em Farmácia pela Faculdade Independente do Nordeste. Email: naysnan17@ hotmail.com

${ }^{2}$ Farmacêutica Bioquímica graduada pela Universidade Tiradentes, Especialista em Análises Clínicas pela Universidade Castelo Branco e docente da Faculdade Independente do Nordeste.

Email: alanascarvalho@gmail.com

${ }^{3}$ Enfermeira graduada pela Faculdade Independente do Nordeste, pós graduanda em Gestão da Clínica pelo IEP-

Hospital Sírio Libanês. Email: c.amaralmoreno@outlook.com
} 


\section{Introdução}

De acordo com WHO (2012), o parasitismo intestinal ainda é considerado um grave problema de saúde pública, estima-se que mais de 2 bilhões de pessoas estejam infectadas por parasitas intestinais, dentre eles destaca-se: Ascaris lumbricoides, Trichuristrichiura, Necator americanus e Ancylostoma duodenale. A maior concentração dos casos de parasitismo ocorre em áreas onde o saneamento básico é inexistente ou insuficiente, características de países em desenvolvimento com inadequada infra-estrutura sanitária e ambiental (NAVONE et al., 2017).

Barbosa e Vieira (2014, p. 02) salientam que as infecções por geo-helmintíases ainda são negligenciadas e concentram-se "nas populações mais carentes de recursos ligados à educação e saúde, e muitas delas não apresentam altas taxas de mortalidade, embora apresentem alta taxa de morbidade". Desta forma, o impacto do parasitismo intestinal na saúde e na qualidade de vida da população infectada é atribuído principalmente ao seu caráter crônico e insidioso e não à sua mortalidade.

Os infantes em idade escolar e pré-escolar possuem as maiores cargas de enteroparasitoses e é a principal fonte de contaminação ambiental. As crianças estão mais expostas e susceptíveis a infecção, pois elas estão em frequente contato com os agentes causadores de parasitismo, "e também porque, quando realizam sua higiene pessoal sozinhas, esta pode não ser satisfatória”.As infecções de alta intensidade, poliparasitismo, são responsáveis por diversos prejuízos, principalmente deficiências físicas e cognitivasque são observadas em casos de parasitoses que espoliam micronutrientes e vitaminas, causando doenças como a anemia ferropriva (SANTOS et al., 2014, p. 333; WHO, 2012).

Atualmente, devido diversas características do mercado de trabalho, as creches comunitárias tornam-se ambientes comuns para as crianças, que passam boa parte da infância ali. Ressalta-se que o ambiente da creche é um fator de risco para aquisição de parasitas intestinais, pois diversos estudos mostram a alta incidência desse tipo de contaminação em crianças, devido a presença de contaminantes de origem fecal nos fômites e no espaço de convivência das crianças (FIGUEIREDO e QUEROL, 2011; BARBOSA; VIEIRA, 2014; SANTOS et al., 2014).

Assim, considerando as questões que envolvem a contaminação em crianças e a alta incidência de parasitas nesta população, percebe-se a importância de realizar estudos epidemiológicos, acerca do aumento significativo dos casos de parasitoses em crianças com idade pré-escolar, uma vez que para o desenvolvimento de medidas preventivas, os dados serão 
de grande valia. Sendo assim torna-se relevante realizar este estudo a fim de conhecer as necessidades de saúde, com objetivo de crescer a agenda para o revigoramento de políticas públicas voltadas a saúde infantil, bem como ampliar os dados teóricos do tema.

Nesse sentido este estudo teve a seguinte problemática: Qual o perfil dos contaminantes de origem fecal em fômites de uma creche? Qual o local onde foram encontrados mais contaminantes fecais e quais foram os mais prevalentes? E teve como objetivo geral: Identificar e avaliar o perfil epidemiológico de contaminantes de origem fecal, presentes em fômites de uma creche.

\section{Metodologia}

Trata-se de um estudo epidemiológico de caráter transversal onde possui uma abordagem quantitativa. $\mathrm{O}$ trabalho foi realizado em uma creche municipal do interior da Bahia a partir da coleta de amostras em 8 lugares diferentes presentes na parte interna da creche. Foram coletadas amostras em duplicata dos seguintes locais: mesas de atividades, botão de descarga, tampa do vaso, tapete recreativo, brinquedos compartilhados na área de lazer e trocadores. Cabe ressaltar que a coleta foi realizada no turno vespertino, antes da higienização de todos os ambientes e na ausência das crianças. Outra informação relevante é que a creche funciona em período integral, atende 28 crianças que a frequenta diariamente de segunda a sexta-feira.

O local escolhido para realização do estudo foi uma creche municipal, situada no distrito de Lucaia, município de Planalto - BA. O município de Planalto está localizado na microrregião Sudoeste, no estado da Bahia. A população do município, de acordo com o Instituto Brasileiro de Geografia e Estatística - IBGE - a população estimada é de 26.915 habitantes em 2017. Já a população do distrito de Lucaia, segundo o IBGE é de cerca de 9.000 mil habitantes e é importante lembrar que se trata de uma localidade que não tem plano municipal saneamento básico e esgotamento sanitário.

Para auxiliar a coleta adequada das amostras foi utilizado roteiro norteador baseado da técnica de Graham. Esse método foi proposto há cerca de 70 anos, com enfoque em diagnóstico de Enterobius vermiculares. Nesse método é utilizada uma fita adesiva transparente (Durex®), no qual esta é aplicada cinco vezes na mesma superfície, o comprimento do adesivo não deve ultrapassar5 ou 6 centímetros. Após a aplicação e retirada da fita, a mesma deve ser fixada a 
lamina microscópica, com as duas extremidades delimitadas por outra fita adesiva na cor branca, local em que será feita a identificação da amostra. A análise das amostras foi feita no laboratório da Faculdade Independente do Nordeste (FAINOR), para tal foi necessária utilização de microscópio óptico, com aumento de 10x e 40x, não sendo utilizado nenhum tipo de corante.

Após análise das lâminas e obtenção dos resultados laboratoriais, os dados foram organizados e tabulados com auxílio do programa Microsoft Excel 2010.

\section{Resultados e Discussão}

As amostras foram coletadas individualmente em duplicata, em cada objeto selecionado utilizando fita individual para cada tipo de objeto, em seguida, as amostras foram compiladas segundo o local de coleta, e por fim na sequência foi realizado um pool de cada objeto - sendo analisadas, no total, 16 lâminas. Destas, $14(87,5 \%)$ revelaram-se positivas e $2(12,5 \%)$ negativas, como visualiza-se no gráfico 1. Após a negatividade das duas lâminas referentes à tampa do vaso, foi realizada novamente a coleta de novas amostras em duplicata para confirmação do resultado, totalizando assim 18 lâminas ao final do processo.

Gráfico 1 - Frequência relativa (FR\%) do total de elementos positivos e negativos para algum tipo de estrutura parasitária presente em fômites de uma creche do interior da Bahia.

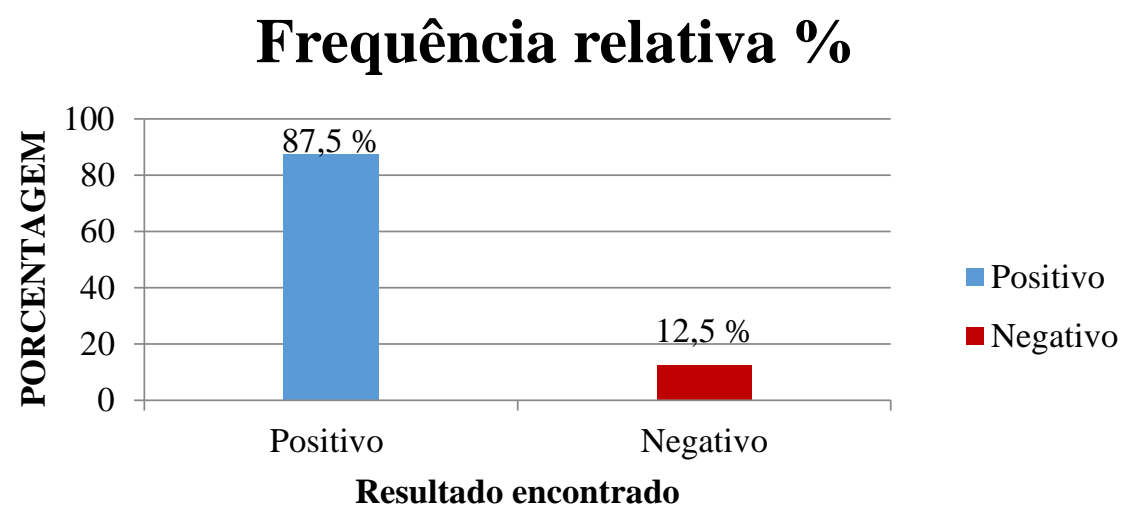

Fonte: Dados da pesquisa, 2017. 
A tabela 1 traz os locais analisados e a quantidade de objetos que participaram da coleta. Como mencionado anteriormente, as tampas dos vasos tiveram sua negatividade confirmada após nova análise para confirmação do resultado.

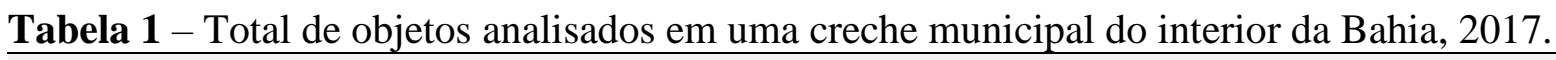

\begin{tabular}{ll}
\hline Objetos analisados & Quantidade de objetos \\
\hline Mesa de atividades (Sala com crianças de 2 anos) & 4 \\
Mesa de atividades (Sala com crianças de 3 anos) & 4 \\
Tampa do vaso & 2 \\
Botão da descarga & 2 \\
Trocador & 3 \\
Tapete recreativo & 1 \\
Brinquedos & 6 \\
Pula Pula & 1 \\
\hline Total -8 & 23 \\
\hline
\end{tabular}

Fonte: Dados da pesquisa, 2017.

O quadro 1 traz as espécies de formas evolutivas encontradas nos objetos analisados. O índice de contaminação foi distinto em todos os locais. Observou-se a maior ocorrência de Enterobius vermiculares em 62,5\% (5) dos locais analisados (M2, M3, BD, T e PP), seguido de os ovos de Ascaris lumbricoides 25\% (2), (T e B), a Fasciola hepática foi encontrada em $25 \%$ (2) dos locais analisados (M2 e B), e seguido de Trichuris trichiura, 12,5\% (1) encontrado apenas no TP, e Strongyloides stercoralis 12,5\% (1), encontrado apenas na M3.

Quadro 1 - Formas evolutivas presentes em objetos analisados em uma creche municipal do interior da Bahia.

\begin{tabular}{|l|l|l|l|l|l|l|l|l|}
\hline Formas parasitárias & M 2 & M3 & TV & BD & T & TP & B & PP \\
\hline $\begin{array}{l}\text { Ovos de Enterobius } \\
\text { vermicularis }\end{array}$ & + & + & - & + & + & - & - & + \\
\hline $\begin{array}{l}\text { Ovos de Trichus } \\
\text { trichiura }\end{array}$ & - & - & - & - & - & + & - & - \\
\hline $\begin{array}{l}\text { Ovos de Ascaris } \\
\text { Lumbricoides }\end{array}$ & - & - & - & - & + & - & + & - \\
\hline $\begin{array}{l}\text { Ovos de Fasciola } \\
\text { Hepática }\end{array}$ & + & - & - & - & - & - & + & - \\
\hline $\begin{array}{l}\text { Larvas de Strongyloides } \\
\text { stercoralis }\end{array}$ & - & + & - & - & - & - & - & - \\
\hline
\end{tabular}

Fonte: Dados da pesquisa, 2017.

M2 = Mesa de atividades (Sala com crianças de 2 anos); M3 = Mesa de atividades (Sala com crianças de 3 anos); TV = Tampa do vaso; $\mathrm{BD}=$ Botão de descarga; $\mathrm{T}=$ Trocador; $\mathrm{TP}=$ Tapete recreativo $; \mathrm{B}=$ Brinquedos $; \mathrm{PP}=$ Pula pula. 
O gráfico 2 traz o de percentual de contaminação dos locais analisados. Observa-se no gráfico que a distribuição percentual foi homogênea em alguns locais como: mesa 2, mesa 3, trocador e brinquedo, que tiveram $40 \%$ de contaminação, seguido do botão da descarga, tapete recreativo e pula pula, com $20 \%$ de contaminação.

Gráfico 2 - Percentual de contaminação pertinente às formas parasitárias encontradas nas lâminas colhidas em objetos analisados em uma creche municipal do interior da Bahia.

\section{\% de contaminação por elementos analisados}

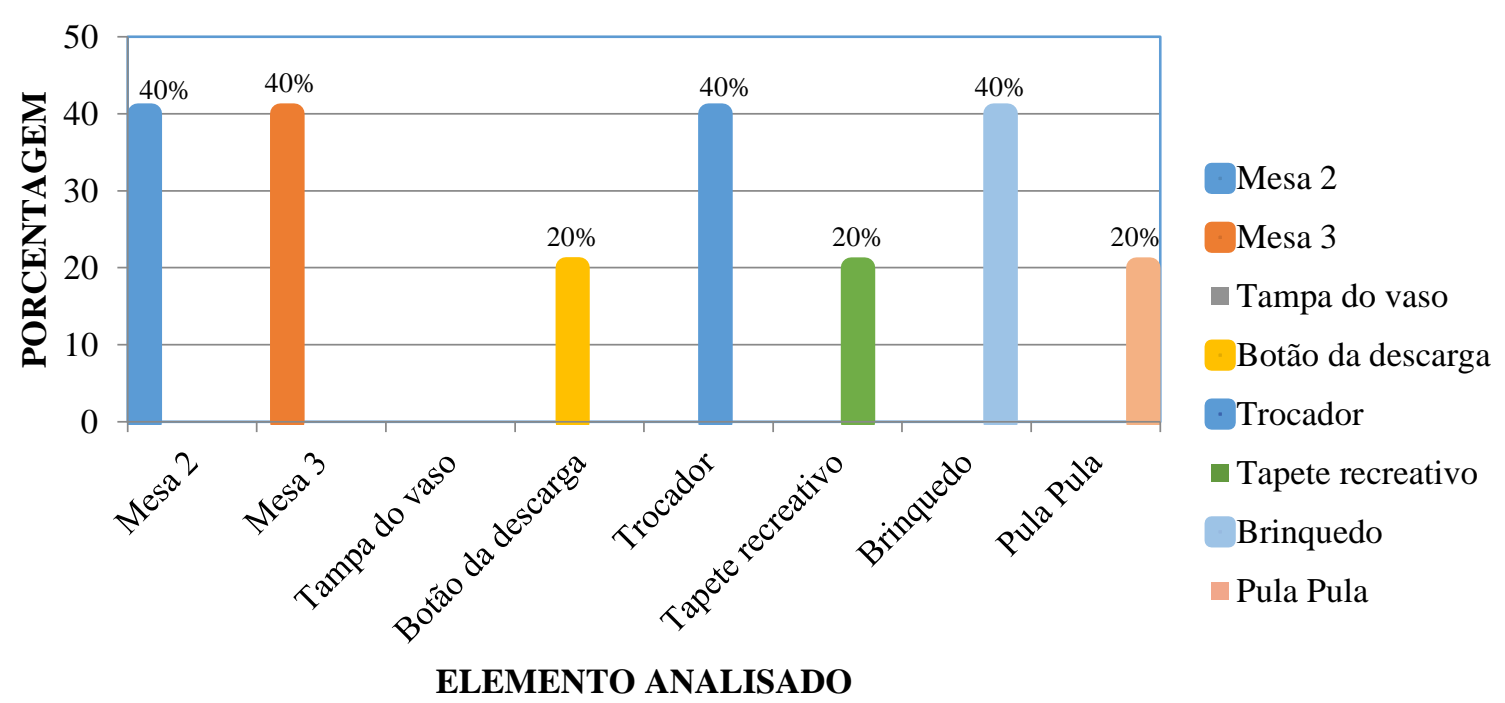

Fonte: Dados da pesquisa, 2017.

De acordo Vitorino et al (2015), o Enterobius vermicularis é o parasita mais cosmopolita que existe entre todos os nematódeos, com prevalência de cerca de $6 \%$ no Brasil por estar associada diversos fatores (culturais, sociais e ambientais), o que se configura uma das razões para a alta incidência dos ovos deste parasita encontrado neste estudo, como mostra o quadro 1, quando comparado aos outros parasitas. Um estudo realizado por Tedescoet al (2012), de cunho transversal, com 242, crianças matriculadas em onzes creches traz resultados semelhantes ao deste estudo, com alta prevalência de nematódeos como Enterobius vermicularis e Ascaris lumbricoides.

As crianças constituem o grupo populacional mais acometido pelas parasitoses intestinais e mais suscetível à elas, haja vista as poucas noções de higiene incutidas por elas, 
bem como pela aglomeração e realização de atividades de lazer em locais coletivos, onde a probabilidade para infecção e reinfecção é alta (BARBOSA e VIEIRA, 2014).

A enterobiose ou oxiurose consiste em uma parasitose singular nos aspectos tangentes a oviposição da fêmea e a dificuldade de encontrar os ovos em exames coprológicos cotidianos. Apesar de ser uma parasitose comum, as complicações causadas pela enterobíase são diversas, mas principalmente as afecções ectópicas para locais como intestino, uretra e ovários. As afecções ectópicas são raras, porém a literatura já descreveu casos no Brasil e também na Europa. Um estudo realizado por Fleminget al (2014), num hospital pediátrico na Irlanda com 182 crianças, verificou que a ocorrência de $7 \%$ dos casos de apendicite aguda foram, causados por Enterobius vermicularis. Destaca-se também que a transmissão dos ovos de Enterobius vermicularis ocorre de forma direta de individuo para indivíduo e não necessita do solo como outros parasitas (TEIXEIRA, et al, 2012)

O segundo enteroparasita mais prevalente deste estudo foi o Ascaris lumbricóides (25\%), assim como em estudos realizados na Universidade do Oeste de Santa Catarina, que encontrou 19 endoparasitas distintos, em que o Ascaris lumbricoides apresentou $20 \%$ de ocorrência. Monteiro et al (2009) verificou em seu estudo de análise coprológica, que os esses ovos também apareceram em 37\% dos pacientes (COSTA, et al 2015; MONTEIRO et al 2009).

Já em relação a um estudo de análise fecal realizado em uma creche por Figueiredo e Querol (2011), mostrou que o índice de Trichuris trichiura foi de 6 (16.22\%), outro estudo, realizado por Monteiro et al (2009), também apresentou resultados com altos índices de Trichuris trichiura $(21,6 \%)$, acima do resultado aqui encontrado que foi de $12,5 \%$.

Outro parasita que merece destaque é Fasciola hepática, que foi encontrada em $25 \%$ (2), dos locais analisados. De acordo Gulhanet al, (2014), a contaminação por Fasciola hepática, pode levar a danos graves para as crianças infectadas. Trata-se de uma parasitose que infecta não só os humanos, mas diversos mamíferos como: bovinos, caprinos. Sua transmissão se dá através de alimentos contaminados e também através de carcaças de animais contaminados.

Em relação ao Strongyloides Stercoralis, verificou-se ocorrência de larvas do mesmo em 12,5\% (1),dos objetos analisados. De acordo Antunes et al (2017), parasitas como Strongyloides Stercoralis são encontrados em baixo percentual, por conta do ciclo biológico do parasita, os ovos ou as larvas necessita da terra/solo para o desenvolvimento. O estudo de Souza et al (2016), também realizado em uma creche encontrou resultado semelhante ao do presente estudo, com a presença da larva de Strongyloides stercoralis em apenas 1 local. Cabe pontuar 
ainda que, de acordo Scharet al, (2013) trata-se de um parasita negligenciado, com poucos estudos acerca do mesmo.

Dentre os fatores que contribuem para a alta prevalência de endoparasitas em creches, assim como a transmissão entre as crianças, se deve ao fato da infra-estrutura inadequada, noções de higiene nulas por parte das crianças, alto número de crianças que frequentam o ambiente, higienização feita de maneira incorreta nos alimentos e locais e objetos utilizados pelas crianças. Além destes fatores, a ausência de plano de saneamento básico, contribui para a transmissão de enteroparasitas de forma significativa. (RODRIGUES et al 2016; VITORINO et al, 2015).

A ocorrência de ovos e larvas dos parasitas verificados pelos dados apresentados no quadro e gráficos da pesquisa houve também a confirmação da vice-diretora da creche que relata que as crianças realmente apresentam sintomatologia compatível com verminoses. É extremamente importante que o diagnóstico das enteroparasitoses seja ágil, uma vez que a presença de sinais e sintomas é mais comum quando o indivíduo apresenta carga parasitária elevada, especialmente em crianças pré-escolares. Dentre os sintomas mais comuns, está a presença de diarréia constante ou intermitente, dor abdominal manchas esbranquiçadas, falta de apetite e distensão abdominal (ANTUNES; LIBARDONI, 2017).

É importante lembrar que ainda hoje, existe negligência acerca das infecções parasitárias por ancilostomídeos e outras helmintíases intestinais. Estas doenças, centralizadas nas populações com baixas condições socioeconômicas, sustentado a tendência epidemiológica de algumas décadas atrás (BARBOSA e VIEIRA, 2014).

\section{Conclusão}

Com base nos dados levantados pela pesquisa e nas discussões levantadas acima, verifica-se que a incidência de parasitas em creches é um problema de saúde pública de alta magnitude que merece atenção especial, haja vista as implicações na saúde das crianças devido às enteroparasitoses.

Ressalta-se que o melhoramento da infra-estrutura da creche, além do estimulo a medidas de limpeza e capacitação dos funcionários, contribuem para disseminação dos parasitas, especialmente do Enterobius vermicularis, que apresentou maior ocorrência em 
diversos locais. Além disso, a execução de um plano de saneamento básico traria contribuições importantes para diminuição das enteroparasitoses.

Por fim, conclui-se que este trabalho atingiu ao objetivo proposto inicialmente, que foi de identificar e avaliar o perfil epidemiológico de contaminantes de origem fecal, presentes em fômites de uma creche. Em relação à hipótese levantada anteriormente, verifica-se que há uma prevalência de contaminantes fecais, significativamente alta. Destaca-se ainda, que a realização de outros estudos como estes é extremamente válido, devido a relevância do tema para a saúde pública e para sociedade.

\section{Referências}

ANTUNES, A. S.; LIBARDONI, K. S. B. Prevalência de enteroparasitoses em crianças de creches do município de Santo Ângelo, RS. Revista Contexto \& Saúde, v.17, n.32,p.:144 156,2017

BARBOSA V.A; VIEIRA de F.O. Educação sanitária como prática de prevenção de parasitoses intestinais em creches. Centro Universitário Metodista Izabela Hendrix; v.unico, $12 \mathrm{p}, 2014$.

COSTA, T. D.; ANDRADE, D. F. R.; BARROS, V. C. B.;FREITAS, D. R. J. Análise de enteroparasitoses em crianças em idade pré-escolar em município de Santa Catarina, Brasil. Rev. Pre. Infec e Saúde, v.1, n.2, p:1-9, 2015.

FIGUEIREDO M.I.O, QUEROL E. Levantamento das parasitoses intestinais em crianças de 4 a 12 anos e funcionários que manipulam o alimento de um centro socioeducativo de Uruguaiana, RS, Brasil. BiodiversidadePampeana PUCRS, Uruguaiana,v.9, n.1, p.3-11, 2011.

FLEMING, C. A.; KEARNEY, D. E.; MORIARTY, REDMOND, H. P.; ANDREWS, E. J. An evaluation of the relationship between Enterobiusvermicularis infestation and acute appendicitis in a paediatric population - A retrospective cohort study.InternationalJournalofSurgery. v.18, p.:154-158, 2015.

GÜLHAN, B.; KANIK YÜKSEK, S.; TEZER, H.; ÖZKAYA PARLAKAY, A.; DALGIÇ, B., DALGIÇ, A.; YILMAZ. Partial Hepatectomy for the Resistant Fasciola Hepatica Infection in a Child.APSP J Case Repv.6, n3, 27p, 2015.

MONTEIRO, A.M.C. Parasitoses intestinais em crianças de creches públicas localizadas em bairros periféricos do munícipio de Aoari, Amazonas, Brasil. Revista de patologia tropical, v.38, n.4, p: 284-290, 2009. 
NAVONE G.T, et al. Estudio transversal de lasparasitosisintestinalesenpoblacionesnfantiles de Argentina.Rev Panam Salud Publica 41, e24, 9p, 2017.

RODRIGUES, T. L.; SILVA, A. S.; SOBRINHO, D. D. T. M; SARAIVA, K. S.; OLIVEIRA, C. P. A. Prevalência de enteroparasitoses em crianças em uma creche do sertão central. Mostra Científica da Farmácia, Centro Universitário Católica de Quixadá, 2016.

SANTOS J, DUARTE A.R.M., GADOTTI G, LIMA L.M. Parasitoses intestinais em crianças de creche comunitária em Florianópolis, SC, Brasil. Rev Patol Trop, v.43, n.3, p.332- 340, 2014.

SCHÄR, F. et al. Strongyloidesstercoralis: Global Distribution and Risk Factors.PLoSNegl Trop Dis. v.11, n.7:e2288, 2013 DOI: 10.1371/journal.pntd.0002288. Print 2013.

TEDESCO, R. M.; CAMACARO, Y.; MORALES, G.; AMAYA, I.; BLANCO, Y.; DEVERA, R. Parásitosintestinalesenniños de hogares de cuidado diariocomunitarios de ciudad Bolívar, estado Bolívar, Venezuela. Oriente, v.24, n.2, p.:142-150, 2012.

TEIXEIRA, E. C.; KOVALICZN, R. A.; BRITO, P. S.Análise de método alternativo para pesquisa de enterobiose. Publ. UEPG Ci. Biol. Saúde, v.18, n.2, p. 109-114, 2012.

VITORINO, R. R.; GOMES, A. P.; FREITAS, R. B.; PEREIRA, S. O.; MOREIRA, T. R.; SANTANA, L. R. Enterobíase: aspectos atuais.Pediatria Moderna, v.51, n.1, p.: 25-29, 2015.

WORLD HEALTH ORGANIZATION (WHO).Eliminating soil-transmitted helminthiases as a public health problem in children.Progress Report 2001-2010 and Strategic Plan 20112020.Geneva: WHO, 2012.

\section{Como citar este artigo (Formato ABNT):}

ALMEIDA, Naysnan R.B.; CARVALHO, Alana S; MORENO, Camila A. Análise Parasitológica de contaminantes de origem fecal encontrados em uma Creche do interior da Bahia. Id on Line Revista Multidisciplinar e de Psicologia, 2017, vol.11, n.38, p.642-651. ISSN: 1981-1179.

Recebido: 06.11.2017

Aceito: 06.11.2017 